\title{
Discovery of Fluorescence Polarization Probe for the ELISA-Based Antagonist Screening of $\alpha_{1}$-Adrenergic Receptors
}

\author{
Zhao Ma, Zhenzhen Liu, Tianyu Jiang, Tianchao Zhang, Huateng Zhang, Lupei Du, and Minyong Li* \\ Department of Medicinal Chemistry, Key Laboratory of Chemical Biology (MOE), School of Pharmacy, Shandong University, Jinan, \\ Shandong 250012, China
}

\section{Supporting Information}

\begin{abstract}
High-throughput screening (HTS) of ligand library to find new active molecules for $G$ protein-coupled receptors is still a major interest, as well as an actual challenge. Fluorescence polarization (FP) assay portrays an essential role in HTS; however, in many cases, it was restricted by the absence of FP probes, the narrow measurement window, and low signal-to-noise $(\mathrm{S} / \mathrm{N})$ ratio. Herein, based on the modification of our previous probe 1 (QFL), we discovered an FP probe 3 (QGGFL) for $\alpha_{1}$-adrenergic receptors $\left(\alpha_{1}\right.$ ARs), which has satisfactory fluorescence intensity, specific

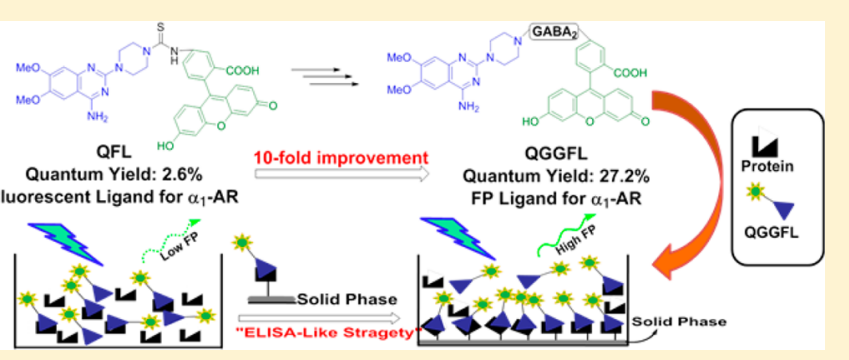
binding ability to receptors, and suitable fluorescence properties that were compatible with the filters in the FP system. Meanwhile, an "ELISA-like" strategy was designed for FP-based HTS assay in which proteins were adhered into a solid phase to improve the measurement window and S/N ratio. With fluorescent antagonist QGGFL and the ELISA strategy, we succeeded in establishing the first competitive binding FP assay for $\alpha_{1}$-AR antagonists as the alternative of the radioligand binding assay.
\end{abstract}

KEYWORDS: $\alpha_{1}$-Adrenergic receptors, fluorescence polarization, ligand screening, fluorescent probe, ELISA, GPCR

G protein-coupled receptors (GPCRs) represent the largest family of transmembrane proteins in the human genome that account for about $40 \%$ of therapeutic agents in the prescription pharmaceutical industry. ${ }^{1,2}$ Unbiased identification of compounds interacting with GPCRs is strongly dependent on highthroughput screening (HTS) of chemical libraries. Therefore, developing analytical methods to find new lead compounds targeting GPCRs is a great interest, as well as an actual challenge. ${ }^{3}$ Currently, HTS approaches for GPCR ligands mainly involve the ligand-receptor binding assay using radioactive or fluorescent tracers. ${ }^{4}$ Radioligand binding is a well-established tool for screening at GPCRs, but with drawbacks of safety concerns and costly consumables. ${ }^{5}$ In parallel to the rapid development of fluorescent techniques, fluorescence assay is becoming a multifaceted tool in life science. ${ }^{6}$ Among these techniques, the nonradioactive, low-cost, and self-referencing FP assay using fluorescent ligands propose another credible alternative. ${ }^{7,8}$ Polarization value is defined as the ratio of fluorescence intensities parallel $\left(I_{\|}\right)$and perpendicular $\left(I_{\perp}\right)$ with respect to the plane-polarized excitation light. ${ }^{9,10}$ In general, fast rotation of freely moving fluorophores will scramble polarization, while constrained fluorophores that bind to bulk receptors will emit highly polarized fluorescence.

Fluorescent ligands of GPCRs sprang up in recent decades, but their applications were restricted primarily to receptor visualization and localization. ${ }^{11,12}$ Although fluorescent ligandbased assay has opened a brand new standpoint in the fields of GPCRs, it still requires further development. ${ }^{13}$ Due to the absence of FP probes, the narrow measurement window, and low signal-to-noise $(\mathrm{S} / \mathrm{N})$ ratio, $\mathrm{FP}$ assays have not been extensively applied. ${ }^{13}$ The key issue in such a matter is that the polarization is not totally or completely inexistent when fluorescent ligands bind to GPCRs because there is not a difference in rotation rate between the free fluorescent ligand and ligand confined by the receptor.

$\alpha_{1}$-Adrenergic receptors ( $\alpha_{1}$-ARs) are members of GPCR superfamily that mediate many physiological processes such as smooth muscle contraction, myocardial inotropy and chronotropy, and hepatic glucose metabolism. ${ }^{14}$ As therapeutic targets, the antagonists of $\alpha_{1}$-AR play critical roles in the treatment of hypertension, benign prostatic hyperplasia $(\mathrm{BPH})$, and lower urinary tract symptoms (LUTS). Moreover, some $\alpha_{1^{-}}$ adrenoceptor antagonists could induce prostate cancer cell apoptosis. ${ }^{15,16}$ However, the screening of $\alpha_{1}$-AR antagonists is heavily dependent on radioligand binding assay at present. ${ }^{17,18}$ Although several fluorescent probes have been synthesized to study $\alpha_{1}$-ARs in cells, a few of them are qualified for the highthroughput screening of antagonists, and no FP-based assay has been discovered or reported. ${ }^{18-22}$ In this note, through the structure modification of our previous fluorescent antagonist QFL (1) of $\alpha_{1}-\mathrm{AR}^{21}$ we designed and synthesized an excellent FP antagonist probe QGGFL (3) with maintained binding affinity and increased fluorescence quantum yield $(\sim 10$-fold $)$,

Received: February 1, 2016

Accepted: August 26, 2016

Published: August 26, 2016 
which could serve as an efficient toolkit to achieve an FP-based HTS assay (Figure 1). To settle the matters of narrow

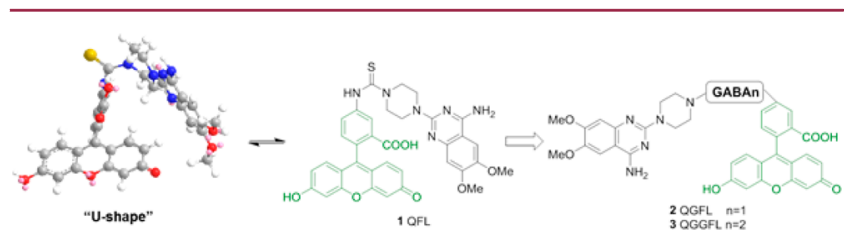

Figure 1. Chemical structure, U-shaped preferential conformation, and structure modification strategy of our previous fluorescent ligand 1 (QFL) of the $\alpha_{1}$-AR.

measurement window and low $\mathrm{S} / \mathrm{N}$ ratio in $\mathrm{FP}$ assay, an "ELISA-like" strategy was designed to amplify FP effect. ${ }^{23,24}$ This strategy is to adhere $\alpha_{1}$-AR proteins to the solid phase and then to measure the FP effect of fluorescent ligands binding to these proteins. When bound to the confined solid-phase proteins, the rotation of fluorescent ligands was constrained greatly, which would produce a considerable polarization effect.

To achieve the FP-based ligand binding assay, the first thing we need to do is to afford an excellent FP probe with bright fluorescence and high binding affinity to receptors. In particular, the fluorescence intensity must be strong enough in order to increase $\mathrm{S} / \mathrm{N}$ ratio and measure up to the microplate reader. Among masses of fluorophores, fluorescein performs brightly as the signal emitter in FP assay by virtue of its high quantum yield. ${ }^{25,26}$ However, in our previous research on $\alpha_{1}$ $\mathrm{AR}$, the reported fluorescent quinazoline-fluorescein conjugate QFL (1, Figure 1), possessing nanomolar binding affinity to $\alpha_{1}$ ARs, showed very low quantum yield (Table 1$).{ }^{21}$ As a result,

Table 1. Characterization of Fluorescent Ligands in Spectroscopy and Binding Affinity to $\alpha_{1}$-ARs

\begin{tabular}{lcccccc} 
& & & & \multicolumn{4}{c}{$K_{\mathrm{i}}^{a}(\mathrm{nM})$} \\
\cline { 5 - 7 } \multicolumn{1}{c}{ compd } & $\lambda_{\text {ex }}(\mathrm{nm})$ & $\lambda_{\mathrm{em}}(\mathrm{nm})$ & $\Phi(\%)$ & $\alpha_{1 \mathrm{~A}}$ & $\alpha_{1 \mathrm{~B}}$ & $\alpha_{1 \mathrm{D}}$ \\
phentolamine & & & & 1.3 & 7.7 & 13.8 \\
$\mathrm{QFL}^{21}$ & 485 & 517 & 2.6 & 4.7 & 7.3 & 20.7 \\
2 & 500 & 530 & 17.1 & 33.5 & 8.1 & 31.3 \\
3 & 500 & 530 & 27.2 & 4.0 & 2.7 & 23.8 \\
atropine & & & & $\mathrm{NA}$ & $\mathrm{NA}$ & $\mathrm{NA}$
\end{tabular}

${ }^{a} K_{\mathrm{i}}$ was calculated from $\mathrm{IC}_{50}$ using the Cheng-Prusoff equation (eq S3).

this compound failed to be employed in quantitative screening of $\alpha_{1}$-AR antagonists and could not become an FP probe. ${ }^{21}$ With the help of Chemdraw 3D software, we found QFL's essential conformation with a crowded U-shape (Figure 1). In such a conformation, the quinazoline group comes close to the fluorescein core, which may exert an influence on the conjugate plane of fluorophores, and thereby the quantum yield decreases. While the quinazoline moiety represents an interesting pharmacophoric scaffold of antagonist to develop FP agents that bind to the $\alpha_{1}$-AR receptor tightly, to circumvent the issue about fluorescence intensity, two novel fluorescent ligands, QGFL and QGGFL (2 and 3, Figure 1), were designed by introducing the linker, $\gamma$-aminobutyric acid (GABA), to conjugate pharmacophore and fluorophore. Although GABA is the inhibitory neurotransmitter targeted at GABA receptors, the new compounds including GABA linker here will show no agonism activity to GABA receptors because the primary amino group at 4-position in GABA is necessary for its agonism activity. ${ }^{27}$ As the linker, GABA could increase the flexibility of molecules and reduce the steric hindrance between quinazoline and fluorescein groups. The target compounds were conveniently synthesized as depicted in Scheme 1 , in which piperazine-substituted chlorine of $\mathbf{6}$ afforded compound 7 , and then the amide condensation and N-Boc deprotection reactions were involved to introduce one or two GABA(s) to the key intermediates 9 and 10. Finally, the key intermediate reacted with FITC to provide 2 (QGFL) and 3 (QGGFL), respectively.

The affinities of two target compounds 2 (QGFL) and 3 (QGGFL) were assessed routinely by competitive binding assays on three $\alpha_{1}$-AR subtypes stably expressed in CHO cells, using radiolabeled $\left[{ }^{3} \mathrm{H}\right]$ prazosin, an established $\alpha_{1}$-AR competitive antagonist. As expected, each compound revealed up to nanomolar affinities for all $\alpha_{1}$-AR subtypes (Table 1 ). This result also indicated that the new compounds could serve as antagonists of $\alpha_{1}$-AR. It should be noted that our previous research indicated clearly that the large moiety was permitted to conjugate to the $\mathrm{N} 4$ position of 2-piperazine group in the quinazoline pharmacophore without losing much binding affinity, but bulk fluorescein was not under consideration for selection. $^{21}$ These results revealed that introducing a linker between fluorescein and quinazoline groups was beneficial for affinity improvement. Moreover, compound 3 (QGGFL) with the longer linker showed higher affinity than compound 2 (QGFL).

A summary of spectral properties for all compounds is shown in Table 1 as well. Compared to the reported compound $\mathbf{1}$ (QFL), the newly synthesized fluorescent ligands 2 (QGFL) and 3 (QGGFL) displayed about $15 \mathrm{~nm}$ red-shift in wavelength. Significantly, the major improvement in optical properties among them was fluorescence quantum yield, and the quantum yield was positively associated with the length of the linker. In brief, compound 3 (QGGFL) with two GABAs as the linker displayed about 10 -fold quantum yield $(27.2 \%)$ than compound 1 (QFL) without linker (2.6\%), and compound 2 (QGFL) with one GABA linker showed 6-fold yield (17.1\%).

An essential requirement of a fluorescent ligand of GPCR is to visualize the target protein. As an improved fluorescent ligand, compound 3 (QGGFL) shows the reasonable receptor affinity and appropriate quantum yield, especially the latter. Next, we established the transfected HEK293A cells transiently with $\alpha_{1 \mathrm{~B}}-\mathrm{AR}$ and determined the ability of QGGFL to bind to such intact cells expressing $\alpha_{1}$-AR in culture. In confocal imaging study, the $\alpha_{1 \mathrm{~B}}$-AR transfected HEK293A cells could be "dyed" by strong green fluorescence when incubated with 300 $\mathrm{nM}$ compound 3 (QGGFL) for $20 \mathrm{~min}$, and the strong fluorescence would weaken while a coincubation of QGGFL $(300 \mathrm{nM})$ and an excess of prazosin $(3 \mu \mathrm{M})$ was carried out (Figure S3). Meanwhile, the untransfected HEK293A cells were also employed as a negative control in which very weak fluorescence was observed. This negative result indicated that nonspecific binding is absent between QGGFL and cell components. The untransfected HEK293A cells also express some $\beta$-ARs natively, so these imaging results also indicated that QGGFL showed no interactions with the aminergic $\beta$-ARs. As is known, the $\alpha_{1 \mathrm{~B}} \mathrm{AR}$ subtype mainly distributes on cytomembrane. ${ }^{28}$ In our confocal imaging, most cells with highlighted membrane by QGGFL came into view, indicative of a cell surface localization for $\alpha_{1 \mathrm{~B}}-\mathrm{AR}$, which was highly consistent with the conclusion from Piascik and declared that the probe 3 (QGGFL) could recognize $\alpha_{1}$-adrenoceptors 
Scheme 1. Synthesis of Fluorescent Ligands 2 and 3

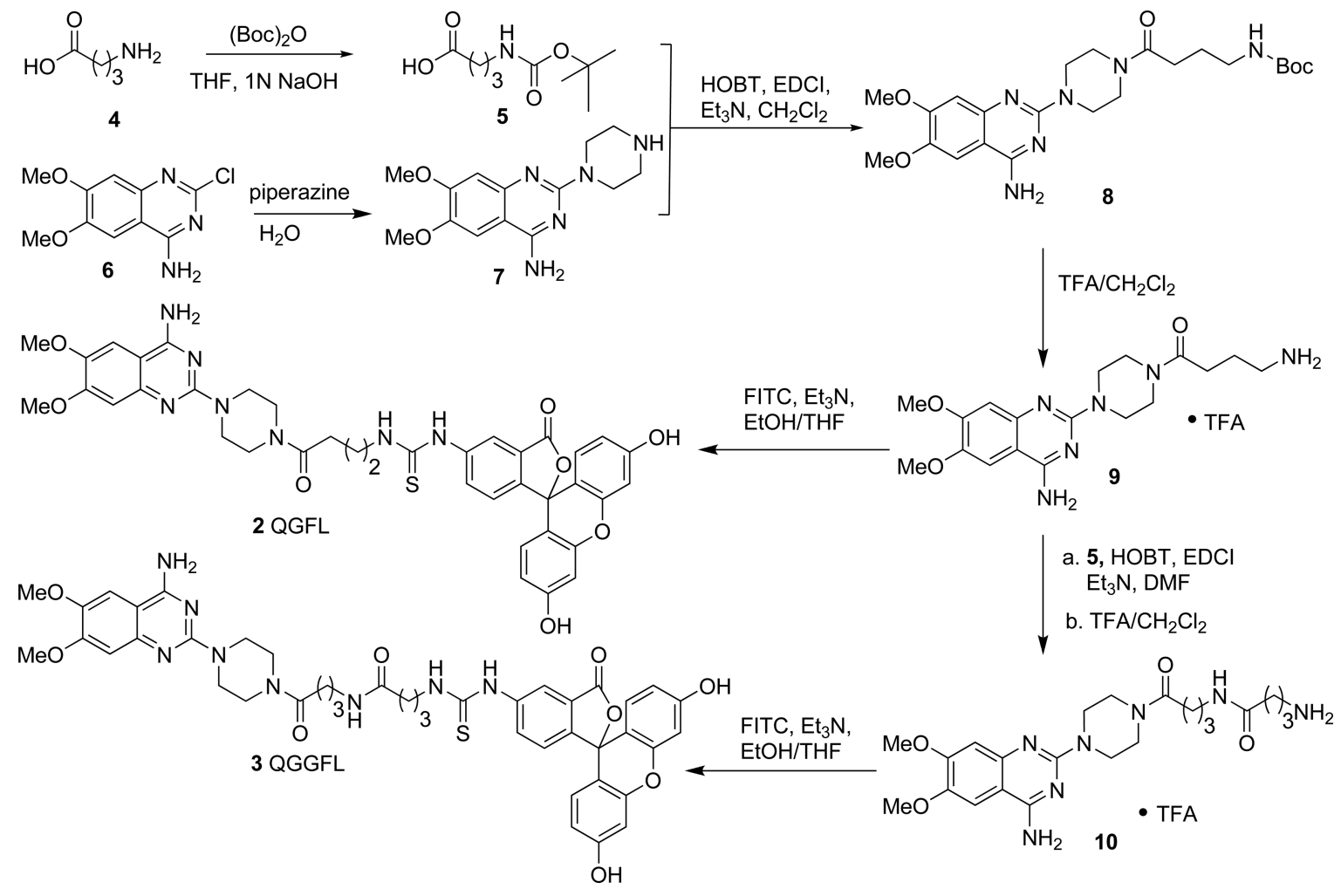

specifically. ${ }^{28}$ In addition, to check the specificity or selectivity of QGGFL, we measured its activities to three kinases (BMX, KIT, and ALK) because quinazoline may cause the inhibitory activity against kinases. ${ }^{29}$ As shown in Figure S5, we found all the $\mathrm{GI}_{50}$ values were greater than $10 \mu \mathrm{M}$. Moreover, QGGFL also showed low affinities to other adrenergic receptors $\left(\alpha_{2}\right.$ and $\beta_{1}$-ARs, Figure S6). As a result, we can conclude that this fluorescent antagonist QGGFL with quinazoline core has few off-target activities.

At present, we have gotten an excellent fluorescent ligand 3 (QGGFL) with improved fluorescence intensity, the specific binding ability to $\alpha_{1}$-AR, and suitable fluorescent properties that were compatible with the filters in our FP system. In order to identify whether the probe QGGFL may serve as an FP probe or if it could be qualified for the FP-based ligand screening of $\alpha_{1}$-AR, polarization at $520 \mathrm{~nm}$ of QGGFL with various concentrations of $\alpha_{1}$-AR proteins was measured first using the 96-well or 384-well no-binding flat bottom black plate (Corning, USA) in classical FP assay. However, as shown in Figure S4Aa, the polarization values seemed to have no correlation with the amount of proteins. This set of irregular data incubated that the measurement window was too narrow for FP assay. To amplify the polarization signal, the ELISA strategy was surrogated (Figure S4B), in which the enzyme or antigen was attached to the solid-phase surface. ${ }^{23,24}$ By adhering to stationary phase, the constrained proteins could slow the spin of fluorescent ligands down, and thus, the fluorescent ligand would produce the obvious polarization effect. To achieve this strategy, 384-well flat bottom black plate with high protein-binding force (Fluotrac 600, Greiner) was employed in $1 \mathrm{~h}$ incubation at room temperature. By contrast with the irregular data in Figure S4Aa, three nice saturation curves in Figure S4Ba showed that addition of $16 \mathrm{nM}$ QGGFL into increasing concentrations of three $\alpha_{1}$-AR proteins gave a saturable increase in FP. We also measured the $\mathrm{Z}^{\prime}$ factor in both the traditional assay and the ELISA-like assay (Figure S4b), which is a statistical benchmark to judge whether the response in a particular assay is large enough to warrant further attention. $^{30,31}$

The $Z^{\prime}$ factors calculated for our ELISA-based FP assay are $0.84,0.77$, and 0.70 for $\alpha_{1 \mathrm{~A}^{-}}, \alpha_{1 \mathrm{~B}^{-}}$, and $\alpha_{1 \mathrm{D}^{-}} \mathrm{AR}$ proteins, respectively (Figure $\mathrm{S} 4 \mathrm{Bb}$ ), and in the traditional $\mathrm{FP}$ assay, the $Z^{\prime}$ factors are $-0.49,-0.64$, and -0.56 , respectively (Figure $\mathrm{S} 4 \mathrm{Ab})$. A $Z^{\prime}$ factor greater than 0.5 means the assay is considered acceptable for a good HTS, while that less than 0 confirms there is too much overlap between the positive and negative controls for the assay to be used. In addition, when treated with excess membrane proteins $(0.4 \mathrm{mg} / \mathrm{mL})$, the FP value displays a dose-dependent enhancement, which yields $K_{\mathrm{d}}$ value of $0.56 \pm 0.14,1.21 \pm 0.49$, and $0.64 \pm 0.30 \mathrm{nM}$ for $\alpha_{1 \mathrm{~A}^{-}}$,

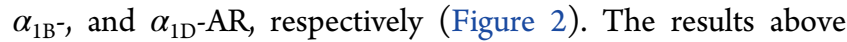
indicate that compound 3 (QGGFL) is qualified to become a FP probe in the evolutionary ELISA-based FP assay. The specific interaction between QGGFL and $\alpha_{1}$-AR proteins reached equilibrium in $60 \mathrm{~min}$ and was saturable. Therefore, the FITC-derivative compound 3 (QGGFL) is suitable for the measurement of binding affinity of ligands.

We further investigated whether this "ELISA-like" FP strategy could be used in HTS for $\alpha_{1}$-AR antagonists. Competitive binding experiments were carried out in the presence of $16 \mathrm{nM}$ QGGFL and $\alpha_{1}$-AR membrane proteins (625 ng of $\alpha_{1 \mathrm{~A}}$-AR and $\alpha_{1 \mathrm{~B}}-\mathrm{AR}, 1.25 \mu \mathrm{g}$ of $\alpha_{1 \mathrm{D}}$-AR, respectively, Table S1). As known competitive antagonists, prazosin, doxazosin, and phentolamine could reduce FP in a concentration-dependent manner, which gave three anticipated sigmoidal response curves (Figure 2) and convincing $\mathrm{IC}_{50}$ and $K_{\mathrm{i}}$ values to three $\alpha_{1}$-AR subtypes with the similar values acquired from radioligand binding assay (Table S2). The 

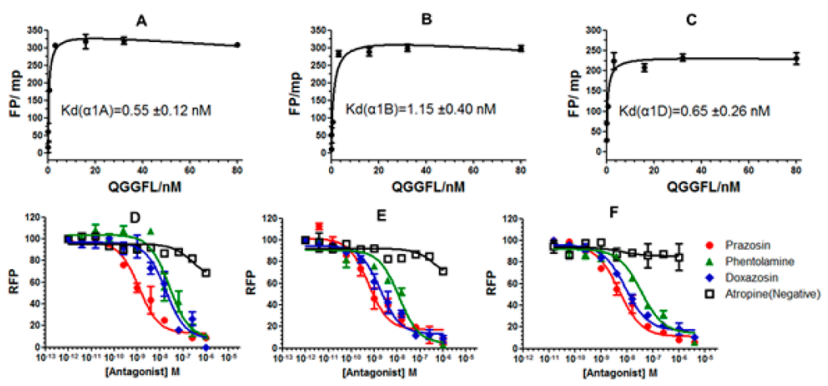

Figure 2. Saturation of increasing concentrations of compound 3 (QGGFL) binding to $\alpha_{1 \mathrm{~A}}-\mathrm{AR}(\mathrm{A}), \alpha_{1 \mathrm{~B}} \mathrm{AR}(\mathrm{B})$, and $\alpha_{1 \mathrm{D}}-\mathrm{AR}(\mathrm{C})$ proteins (final conc. $0.4 \mathrm{mg} / \mathrm{mL}$ ). Competition curves of prazosin, doxazosin, phentolamine, and atropine to three $\alpha_{1}$-AR subtypes, including $\alpha_{1 \mathrm{~A}} \mathrm{AR}(\mathrm{D}), \alpha_{1 \mathrm{~B}} \mathrm{AR}(\mathrm{E})$, and $\alpha_{1 \mathrm{D}} \mathrm{AR}(\mathrm{F})$ using the established "ELISA-like" FP assay.

difference of $K_{\mathrm{i}}$ values in the two assays may be caused by the different systems: the unbound ligands in radioligand assay were washed away, while the FP values were recorded in the homogeneous reaction system. FP was almost enacted on the muscarinic receptor antagonist atropine, which could serve as the negative group for atropine do not bind to $\alpha_{1}$-AR. Comparing with the radioligand competitive binding assay, the safer fluorescent ligands were employed and fewer membrane proteins ( $>10$ fold) were consumed in the FP binding assay (Table S1). According to these results, we also concluded that the new fluorescent antagonist QGGFL could serve as a competitive antagonist.

In conclusion, developing analytical methods to identify compounds interacting with GPCRs or to find new drugs targeting GPCRs is drawing more and more attention from scientists and researchers. Although FP assay can portray important roles in the HTS of the chemical library for GPCRs, it is restricted in many cases due to the lack of FP probes, the narrow measurement window, and low $\mathrm{S} / \mathrm{N}$ ratio. To overcome these limitations in $\alpha_{1}$-ARs, we first discovered and synthesized an ideal FP probe QGGFL, compound 3, for $\alpha_{1}$-ARs, which has improved quantum yield, high receptor affinity, and the ability of receptor imaging specifically in living cells. Moreover, an "ELISA-like" strategy was designed in which proteins are confined to the solid phase in order to amplify the FP signal and increase the measurement window and $\mathrm{S} / \mathrm{N}$ ratio. Based on the FP probe QGGFL and the ELISA strategy, we established a safe and affordable FP-based screening method for $\alpha_{1}$-ARs for the first time, which could provide convincing data of binding affinity to three $\alpha_{1}$-AR subtypes of known antagonists, such as prazosin, doxazosin, and phentolamine, with the classical radioligand binding assay. To the best of our knowledge, this research is the first report that the ELISA strategy is applied in a small-molecule probe-based FP assay. It is our expectation that this strategy would find broad applications in the FP-based HTS for other target proteins to help address their signal issue.

\section{ASSOCIATED CONTENT}

\section{S Supporting Information}

The Supporting Information is available free of charge on the ACS Publications website at DOI: 10.1021/acsmedchemlett.6b00048.

Full experimental procedures, analytical and spectral characterization data of all compounds, and results of cell imaging and FP assay (PDF)

\section{AUTHOR INFORMATION}

\section{Corresponding Author}

*Tel/Fax: +86-531-8838-2076. E-mail: mli@sdu.edu.cn.

\section{Author Contributions}

The manuscript was written through contributions of all authors. All authors have given approval to the final version of the manuscript.

\section{Notes}

The authors declare no competing financial interest.

\section{ACKNOWLEDGMENTS}

The present work was supported by grants from the Qilu Young Professorship of Shandong University, the International Postdoctoral Exchange Fellowship Program, the Fok Ying Tong Education Foundation (No. 122036), the Program for Changjiang Scholars and Innovative Research Team in University (No. IRT13028), the Major Project of Science and Technology of Shandong Province (No. 2015ZDJS04001), the Shandong Key Research \& Development Project (No. 2015GSF118166), and the Fundamental Research Funds of Shandong University (No. 2014JC008). Our cell imaging work was performed at the Microscopy Characterization Facility, Shandong University.

\section{DEDICATION}

This article is dedicated to Professor Lin Xia on the occasion of her 80th birthday.

\section{ABBREVIATIONS}

GPCRs, G protein-coupled receptors; HTS, high-throughput screening; FP, fluorescence polarization; $\mathrm{S} / \mathrm{N}$, signal-tonoise; $\alpha_{1}$-ARs, $\alpha_{1}$-adrenergic receptors; $\mathrm{BPH}$, benign prostatic hyperplasia; LUTS, lower urinary tract symptoms; GABA, $\gamma$ aminobutyric acid; ELISA, enzyme linked immunosorbent assay; EDCI, 1-ethyl-3-(3-(dimethylamino)propyl)carbodiimide hydrochloride; HOBT, 1-hydroxybenzotriazole; FITC, fluorescein isothiocyanate; TFA, trifluoroacetic acid; BMX, bone marrow X kinase; ALK, anaplastic lymphoma kinase; KIT, v-kit Hardy-Zuckerman 4-feline sarcoma viral oncogene homologue

\section{REFERENCES}

(1) Ke, N.; Nguyen, K.; Irelan, J.; Abassi, Y. A. Multidimensional GPCR profiling and screening using impedance-based label-free and real-time assay. In G Protein-Coupled Receptor Screening Assays; Springer: New York, 2015; pp 215-226.

(2) Sengupta, S.; Ye, K.; Scott, A. D.; Niu, B.; Bailey, M. H.; McLellan, M. D.; Wendl, M. C.; Wyczalkowski, M. A.; Ding, L. Sequence and structure-guided approach to identify functional mutations in G-protein coupled receptors. Cancer Res. 2015, 75, $61-61$.

(3) Oueslati, N.; Hounsou, C.; Belhocine, A.; Rodriguez, T.; Dupuis, E.; Zwier, J. M.; Trinquet, E.; Pin, J.-P.; Durroux, T. Time-resolved FRET strategy to screen GPCR ligand library. In G Protein-Coupled Receptor Screening Assays; Springer: New York, 2015; pp 23-36.

(4) Smith, D. S.; Eremin, S. A. Fluorescence polarization immunoassays and related methods for simple, high-throughput screening of small molecules. Anal. Bioanal. Chem. 2008, 391, 1499-1507.

(5) Allen, M.; Reeves, J.; Mellor, G. High throughput fluorescence polarization: a homogeneous alternative to radioligand binding for cell surface receptors. J. Biomol. Screening 2000, 5, 63-69.

(6) Leopoldo, M.; Lacivita, E.; Berardi, F.; Perrone, R. Developments in fluorescent probes for receptor research. Drug Discovery Today 2009, 14, 706-712. 
(7) Banks, P.; Gosselin, M.; Prystay, L. Fluorescence polarization assays for high throughput screening of $\mathrm{G}$ protein-coupled receptors. J. Biomol. Screening 2000, 5, 159-167.

(8) Jones, J. W.; Greene, T. A.; Grygon, C. A.; Doranz, B. J.; Brown, M. P. Cell-free assay of G-protein-coupled receptors using fluorescence polarization. J. Biomol. Screening 2008, 13, 424-429.

(9) Jia, T.; Fu, C.; Huang, C.; Yang, H.; Jia, N. A highly sensitive naphthalimide-based fluorescence polarization probe for detecting cancer cells. ACS Appl. Mater. Interfaces 2015, 7, 10013-10021.

(10) Owicki, J. C. Fluorescence polarization and anisotropy in high throughput screening: perspectives and primer. J. Biomol. Screening 2000, 5, 297-306.

(11) Ma, Z.; Du, L.; Li, M. Toward fluorescent probes for G-proteincoupled receptors (GPCRs). J. Med. Chem. 2014, 57, 8187-8203.

(12) Vernall, A. J.; Hill, S. J.; Kellam, B. The evolving small-molecule fluorescent-conjugate toolbox for class A GPCRs. Br. J. Pharmacol. 2014, 171, 1073-1084.

(13) Cottet, M.; Faklaris, O.; Zwier, J. M.; Trinquet, E.; Pin, J.-P.; Durroux, T. Original fluorescent ligand-based assays open new perspectives in G-protein coupled receptor drug screening. Pharmaceuticals 2011, 4, 202-214.

(14) Piascik, M. T.; Perez, D. M. $\alpha_{1}$-Adrenergic receptors: new insights and directions. J. Pharmacol. Exp. Ther. 2001, 298, 403-410.

(15) Kyprianou, N.; Benning, C. M. Suppression of human prostate cancer cell growth by $\alpha_{1}$-adrenoceptor antagonists doxazosin and terazosin via induction of apoptosis. Cancer Res. 2000, 60, 4550-4555.

(16) Partin, J.; Anglin, I.; Kyprianou, N. Quinazoline-based $\alpha_{1^{-}}$ adrenoceptor antagonists induce prostate cancer cell apoptosis via TGF- $\beta$ signalling and $\mathrm{I} \kappa \mathrm{B} \alpha$ induction. Br. J. Cancer 2003, 88, 16151621 .

(17) Nagatomo, T.; Tsuchihashi, H.; Sasaki, S.; Nakagawa, Y.; Nakahara, H.; Imai, S. Displacement by $\alpha_{1}$-adrenergic agonists and antagonists of ${ }^{3} \mathrm{H}$-prazosin bound to the $\alpha_{1}$-adrenoceptors of the dog aorta and the rat brain. Jpn. J. Pharmacol. 1985, 37, 181-187.

(18) Mackenzie, J. F.; Daly, C. J.; Pediani, J. D.; McGrath, J. C. Quantitative imaging in live human cells reveals intracellular $\alpha_{1}$ adrenoceptor ligand-binding sites. J. Pharmacol. Exp. Ther. 2000, 294, 434-443.

(19) Ma, J.; Hou, Z.; Song, Y.; Wang, L.; Guo, E. Visual and quantitative screening of $\alpha_{1}$-adrenoceptor antagonists in living cells using quantum dots. ACS Comb. Sci. 2014, 16, 155-159.

(20) Morishima, S.; Suzuki, F.; Nishimune, A.; Yoshiki, H.; Akino, H.; Yokoyama, O.; Muramatsu, I. Visualization and tissue distribution of $\alpha_{1 \mathrm{~L}}$-adrenoceptor in human prostate by the fluorescently labeled ligand Alexa-488-silodosin. J. Urol. 2010, 183, 812-819.

(21) Zhang, W.; Ma, Z.; Li, W.; Li, G.; Chen, L.; Liu, Z.; Du, L.; Li, M. Discovery of quinazoline-based fluorescent probes to $\alpha_{1}$-adrenergic receptors. ACS Med. Chem. Lett. 2015, 6, 502-506.

(22) Ma, Z.; Lin, Y.; Cheng, Y.; Wu, W.; Cai, R.; Chen, S.; Shi, B.; Han, B.; Shi, X.; Zhou, Y.; Du, L.; Li, M. Discovery of the first environment-sensitive near-infrared (NIR) fluorogenic ligand for $\alpha_{1}$ adrenergic receptors imaging in vivo. J. Med. Chem. 2016, 59, 21512162.

(23) Elder, P.; Lewis, J. An enzyme-linked immunosorbent assay (ELISA) for plasma testosterone. J. Steroid Biochem. 1985, 22, 635638.

(24) de Camargo, Z. P.; Guesdon, J.; Drouhet, P. E.; Improvisi, L. Enzyme-linked immunosorbent assay (ELISA) in the paracoccidioidomycosis. Mycopathologia 1984, 88, 31-37.

(25) Marholz, L. J.; Wang, W.; Zheng, Y.; Wang, X. A fluorescence polarization biophysical assay for the naegleria DNA hydroxylase Tet1. ACS Med. Chem. Lett. 2016, 7, 167-171.

(26) Johnson, I.; Spence, M. T. The Molecular Probes Handbook, A Guide to Fluorescent Probes and Labeling Technologies; Life Technologies: Eugene, OR, 2010.

(27) Dewey, S. L.; Brodie, J. D.; Ashby, C. R., Jr. By administering a drug that increases central nervous system GABA (4-aminobutyric acid) levels; for example gabapentin, valproic acid, progabide, gamma- hydroxybutyric acid, fengabine, cetylGABA, topiramate, tiagabine, or acamprosate. US Patent US6906099 2015, 2005.

(28) Chalothorn, D.; McCune, D. F.; Edelmann, S. E.; GarcíaCazarín, M. L.; Tsujimoto, G.; Piascik, M. T. Differences in the cellular localization and agonist-mediated internalization properties of the $\alpha_{1}$ adrenoceptor subtypes. Mol. Pharmacol. 2002, 61, 1008-1016.

(29) Ravez, S.; Castillo-Aguilera, O.; Depreux, P.; Goossens, L. Quinazoline derivatives as anticancer drugs: a patent review (2011 present). Expert Opin. Ther. Pat. 2015, 25, 789-804.

(30) Zhang, J.-H.; Chung, T. D.; Oldenburg, K. R. A simple statistical parameter for use in evaluation and validation of high throughput screening assays. J. Biomol. Screening 1999, 4, 67-73.

(31) Lei, Y.; Hu, T.; Wu, X.; Wu, Y.; Bao, Q.; Zhang, L.; Xiang, H.; Sun, H.; You, Q.; Zhang, X. Affinity-based fluorescence polarization assay for high-throughput screening of polyl hydroxylase 2 inhibitors. ACS Med. Chem. Lett. 2015, 6, 1236-1240. 